\title{
Asociación entre submarinismo y trastornos neurocognitivos en personal militar retirado
}

Fernando M. Runzer-Colmenares ${ }^{1,2,3, a}$, Adriana Viñas-Mendieta ${ }^{\text {a }}$, Ruth Vela-Sosa ${ }^{\text {a }}$, Alessandra Vasquez ${ }^{\text {a }}$, Angie Cermeño-Maraví ${ }^{a}$, Enrique Moncada-Mapelli ${ }^{\mathrm{a}}$, Ian Falvy-Bockos ${ }^{5, \mathrm{a}}$, Miguel Cadena-Sanabria ${ }^{6,7}{ }^{\text {, José F. Parodi }}{ }^{1,3}$

\section{RESUMEN}

Objetivo: Determinar la asociación entre el haber practicado submarinismo durante el servicio militar y el desarrollo de trastornos neurocognitivos en adultos mayores.

Materiales y métodos: Estudio analítico retrospectivo, proviene del análisis de la base de datos del estudio: Fragilidad y dependencia funcional en adultos mayores, realizado entre 2010-2015 realizado por el personal del servicio de geriatría del Centro Médico Naval del Perú, en niveles asistenciales ambulatorios. En cuanto a la asociación se usó Chi2, p<0,05 e IC 95 \%, para el modelo de regresión, se utilizó un modelo de Poisson.

Resultados: Se analizaron en total 1020 participantes. 322 (42,42 \%) de los participantes estuvieron en el rango de 71 - 80 años de edad. 240 (31,62 \%) presentaban trastornos neurocognitivos. Los factores asociados a los trastornos neurocognitivos fueron los siguientes: el rango de edad entre 71 - 80 años de edad (RP=1,69; IC95 \%: 1,12 - 2,54), enfermedad cerebro vascular (RP=1,48; IC95 \%: 1,01 - 2,23) y especialidad de submarinismo (RP=2,36; IC95 \%: 1,64 - 3,39). Tener grado militar superior se asoció con menor riesgo (RP=0,62; IC95 \%: 0,43 - 0,89).

Conclusiones: Los pacientes que han practicado el submarinismo tienen un mayor riesgo de padecer trastorno neurocognitivo, sin embargo, se requieren estudios prospectivos adicionales para corroborar dicha asociación.

Palabras clave: Submarineros; trastornos neurocognitivos; adulto mayor (Fuente: DeCS BIREME).

\section{Association between scuba diving and neurocognitive disorders in retired military personnel}

\section{ABSTRACT}

Objective: To determine the association between the exposure to scuba diving during military service and the development of neurocognitive disorders in elderly people.

Materials and methods: A retrospective analytical study, derived from the database analysis of the study "Fragility and functional dependence in older adults" conducted between 2010 and 2015 on an outpatient basis by the staff of the geriatrics service of the Centro Médico Naval del Perú (Peruvian Naval Medical Center). The chi-square test, $\mathrm{p}<0.05$ and 95 $\% \mathrm{Cl}$ were used to establish said association, and the Poisson model was used as regression model.

Results: A total of 1,020 participants were analyzed. Three hundred twenty-two (322 [42.42\%]) participants were between 71 and 80 years of age. Two hundred forty $(240$ [31.62 \%]) had neurocognitive disorders. Factors associated with neurocognitive disorders were: age range between 71 and 80 years of age $(P R=1.69,95 \% \mathrm{Cl}: 1.12-2.54)$, cerebrovascular disease $(P R=$ $1.48,95 \% \mathrm{Cl}: 1.01-2.23)$ and major in scuba diving $(\mathrm{PR}=2.36,95 \% \mathrm{Cl}: 1.64-3.39)$. A higher military rank was associated with a lower risk of developing neurocognitive disorders ( $\mathrm{PR}=0.62,95 \% \mathrm{Cl}$ : 0.43-0.89).

Conclusions: Patients who have practiced scuba diving have a higher risk of developing neurocognitive disorders; however, additional prospective studies are required to confirm this association.

Keywords: Submariners; neurocognitive disorders; aged (Source: MeSH NLM).

1. Universidad San Martín de Porres, Facultad de Medicina Humana, Centro de Investigación del Envejecimiento (CIEN), Lima, Perú

2. Universidad Científica del Sur,Facultad de Ciencias de la Salud. Lima, Perú.

3. Bamboo Seniors Health Services. Lima, Perú.

4. Hospital Central de la Fuerza Aérea del Perú, Departamento de Geriatría. Lima, Perú.

5. Clínica FOSCAL, Departamento de Medicina Interna, Unidad de Geriatría. Bucaramanga, Colombia.

6. Universidad Industrial de Santander (UIS). Bucaramanga, Colombia.

a. Universidad de San Martín de Porres, Facultad de Medicina Humana. Lima, Perú. 


\section{INTRODUCCIÓN}

Actualmente vivimos la llamada transición demográfica, las mejoras en salud, entre otros factores, han traído como consecuencia la disminución de las enfermedades transmisibles y una tendencia al aumento de la prevalencia de las enfermedades no transmisibles ${ }^{(1)}$. En el Perú, se utiliza el indicador AVISA, que evalúa la pérdida de años saludables como consecuencia de muerte prematura (AVP) y por la presencia de la discapacidad asociada (AVD) y el contexto de enfermedad crónica atraviesa un momento difícil, con poca presencia de políticas sostenibles que muestren un efecto beneficioso en la población ${ }^{(2)}$. En la última década, nos hemos enfrentado a enfermedades no transmisibles, básicamente crónicas, con los mayores costes sociales y económicos reportados en la literatura ${ }^{(3,4)}$.

Una de estas enfermedades es la demencia o trastorno neurocognitivo mayor. Cifras para el año 2015 mencionan que 46,8 millones de personas viven con trastornos neurocognitivos (TNC) en el mundo y se proyecta que este número se duplique cada 20 años, pudiendo alcanzar los 74,7 millones en el 2030 y los 131,5 millones en el $2050{ }^{(5)}$. En estudios locales se encontró que la prevalencia de los TNC fue del $9,3 \%^{(6)}$, en otro estudio realizado por el Instituto Nacional de Salud Mental en Lima se encontró una prevalencia de $6,7 \%^{(7)}$. Parodi et al; realizó un estudio en el 2017; la muestra fue de 657 adultos mayores de los cuales el 20,1 \% desarrolló TNC durante el seguimiento ${ }^{\left({ }^{(8)}\right.}$.

Los TNC tienen un impacto sobre la persona, la familia y también la sociedad, se asocian a un aumento de los costos en salud y, en las Américas, el costo para tratar y cuidar a las personas con demencia es alto en relación a otras patologías crónicas ${ }^{(9)}$. Existen diversos factores de riesgo conocidos para sufrir TNC, como hipoxia, enfermedad cardiovascular, enfermedad cerebrovascular y pobre educación ${ }^{(10,11)}$. La mayoría de ellos comparten componentes fisiopatológicos comunes, como la deprivación de oxígeno a nivel cerebral y disminución de la irrigación encefálica ${ }^{(12)}$.

La exposición a ambientes con presiones de oxígeno extremas se asocia a ciertas patologías, en especial cuando se realizan sin el equipamiento adecuado o de manera crónica ${ }^{(13)}$. Las enfermedades más estudiadas son las relacionadas a oído medio o necrosis avascular ósea, entre otras ${ }^{(14,15)}$. Al respecto, existe evidencia de daño del sistema nervioso central: Kay Tetzlaff et al compararon resonancias magnéticas cerebrales de veinte buceadores con al menos veinte horas de experiencia con un grupo sin experiencia previa, observándose en el segundo grupo un mayor número de anomalías, incluso sin clínica neurológica significativa ${ }^{(16)}$. No se ha explorado la relación causal entre el submarinismo como actividad laboral y el desarrollo de TNC, pese a que existen ciertos puntos en común con factores de riesgo conocidos, por lo cual, el objetivo del presente estudio es determinar la asociación entre el haber estado expuesto a submarinismo durante el servicio militar y el desarrollo de TNC en adultos mayores.

\section{MATERIALES Y MÉTODOS}

El estudio proviene del análisis de la base de datos del estudio Fragilidad y dependencia funcional en adultos mayores, realizado entre 2010-2015, llevado a cabo por el personal del servicio de geriatría del Centro Médico Naval del Perú, en niveles asistenciales ambulatorios ${ }^{(17)}$. El diseño del estudio fue analítico retrospectivo. Los criterios de inclusión del estudio fueron: participantes con edad de 60 años o más, sin antecedente de TNC. Se excluyeron 129 participantes por tener un puntaje de MiniMental State Examination (MMSE. Versión 1) ${ }^{(18)}$ menor a 22, y a 131 sujetos por presentar TNC antes del ingreso al estudio. Se usó codificación para proteger la identidad de cada participante. Para la descripción de los datos recolectados se utilizaron gráficas estadísticas y tablas de frecuencia y para el análisis tablas de contingencia. En cuanto al análisis bivariado, se usó la técnica de Chi cuadrado, con un nivel de significancia de $\mathrm{p}<0,05$ e IC $95 \%$; para el modelo de regresión, se utilizó un modelo de Poisson.

\section{Variables}

- Especialidad durante servicio militar: Submarinismo, otros (maquinaria, sanidad, ingeniería, inteligencia, guardacostas, administración, técnicas navales).

- Trastorno neurocognitivo: Definido como la presencia de un puntaje menor a 22 en la escala MMSE durante 2010-2015, en base a un puntaje normal al inicio del seguimiento y ausencia de TNC previo.

- Características sociodemográficas: edad, sexo, según documento de identidad. Educación, categorizada en colegio completo/incompleto y educación técnica superior. Grado militar: superior o subalterno.

- Comorbilidades, definidas según su presencia en la historia clínica: Diabetes mellitus tipo II, hipertensión, enfermedad renal crónica (ERC), depresión, sobrepeso/ obesidad, antecedente de hábito tabáquico, enfermedad cerebrovascular.

- Sedentarismo: definido según la escala Physical Activity Scale for the Elderly (PASE), adaptada al español (19). Se crearon cuartiles (q1, q2, q3 y q4), dicotomizando a la variable de la siguiente manera: Actividad física adecuada: q3 y q4, sedentarismo: q1 y q2.

\section{RESULTADOS}

Análisis descriptivo y bivariado según antecedente de submarinismo durante servicio militar

Se analizaron en total 1020 participantes. $322(42,42 \%)$ participantes del total estuvieron en el rango de $71-80$ años de edad; 587 (77,34 \%) de los participantes contaban con educación escolar entre completa e incompleta. $88(11,59 \%)$ participantes presentaban antecedente de submarinismo. Los participantes con antecedentes de submarinismo padecían en menor porcentaje de diabetes mellitus, enfermedad crónica renal, depresión, sobrepeso u obesidad, enfermedad vascular, hipertensión arterial a comparación de aquellos participantes que no contaban con antecedentes de submarinismo. 
De todas las patologías mencionadas anteriormente se observó que la diabetes mellitus, depresión, sobrepeso u obesidad, resultaron no significativas.

En referencia al hábito tabáquico, $20(4,10 \%)$ de los participantes con antecedente submarinista lo presentaba; sin embargo, en los participantes sin antecedente submarinista era de $468(95,90 \%)$, notándose así una cifra mayor. Durante la realización del estudio y el seguimiento del total de participantes tanto participantes con antecedentes submarinistas como los que contaban con otra especialidad; se encontró que 240 (31,62 \%) de los participantes padecían de TNC, de este total, $162(67,50 \%)$ tenía otra especialidad y $78(32,50 \%)$ contaba con antecedente submarinista (Tabla 1 ).

Tabla 1. Análisis descriptivo y bivariado según antecedente de submarinismo durante servicio militar $(n=1020)$

\begin{tabular}{|c|c|c|c|c|c|}
\hline Variables & $\mathrm{n}$ & $\%$ & $\begin{array}{l}\text { Otras } \\
\text { especialidades } \\
n=671(88,41 \%)\end{array}$ & $\begin{array}{l}\text { Antecedente de } \\
\text { submarinismo } \\
n=88(11,59 \%)\end{array}$ & Valor de $p$ \\
\hline \multicolumn{5}{|l|}{ Edad } & 0,4 \\
\hline 60 - 70 años & 149 & 19,63 & $132(88,59)$ & $17(11,41)$ & \\
\hline 71 - 80 años & 322 & 42,42 & $290(90,06)$ & $32(9,94)$ & \\
\hline$>80$ años & 288 & 37,94 & $249(86,46)$ & $39(13,54)$ & \\
\hline \multicolumn{5}{|l|}{ Educación } & $<0,0001$ \\
\hline $\begin{array}{l}\text { Colegio completo/ } \\
\text { incompleto }\end{array}$ & 587 & 77,34 & $117(68,02)$ & $55(31,98)$ & \\
\hline Técnica/superior & 172 & 22,66 & $554(94,38)$ & $33(5,62)$ & \\
\hline \multicolumn{5}{|l|}{ Grado militar } & 0,4 \\
\hline Subalterno & 613 & 80,76 & $126(86,30)$ & $20(13,70)$ & \\
\hline Superior & 146 & 19,24 & $545(88,91)$ & $68(11,09)$ & \\
\hline \multicolumn{5}{|c|}{ Diabetes mellitus tipo 2} & 0,8 \\
\hline Sí & 128 & 16,86 & $114(89,06)$ & $14(10,94)$ & \\
\hline No & 631 & 83,14 & $557(88,27)$ & $74(11,73)$ & \\
\hline \multicolumn{5}{|c|}{ Enfermedad renal crónica } & $<0,0001$ \\
\hline Sí & 163 & 21,48 & $119(73,01)$ & $44(26,99)$ & \\
\hline No & 596 & 78,52 & $552(92,62)$ & $44(7,38)$ & \\
\hline \multicolumn{5}{|c|}{ Hipertensión arterial } & $<0,0001$ \\
\hline Sí & 446 & 58,76 & $413(92,60)$ & $33(7,40)$ & \\
\hline No & 313 & 41,24 & $258(82,43)$ & $55(17,57)$ & \\
\hline \multicolumn{5}{|l|}{ Depresión } & 0,2 \\
\hline Sí & 151 & 19,89 & $138(91,39)$ & $13(8,61)$ & \\
\hline No & 608 & 80,11 & $533(87,66)$ & $75(12,34)$ & \\
\hline \multicolumn{5}{|l|}{ Sobrepeso/Obesidad } & 0,7 \\
\hline Sí & 171 & 22,53 & $150(87,72)$ & $21(12,28)$ & \\
\hline No & 588 & 77,47 & $521(88,61)$ & $67(11,39)$ & \\
\hline \multicolumn{5}{|c|}{ Antecedente de hábito tabáquico } & $<0,0001$ \\
\hline Sí & 488 & 35,7 & $468(95,90)$ & $20(4,10)$ & \\
\hline No & 271 & 64,3 & $203(74,91)$ & $68(25,09)$ & \\
\hline \multicolumn{5}{|l|}{ Sedentarismo } & $<0,0001$ \\
\hline Sí & 162 & 78,66 & $119(73,46)$ & $43(26,54)$ & \\
\hline No & 597 & 21,34 & $552(92,46)$ & $45(7,54)$ & \\
\hline \multicolumn{5}{|c|}{ Enfermedad cerebrovascular } & $<0,0001$ \\
\hline Sí & 42 & 5,53 & $21(50,0)$ & $21(50,00)$ & \\
\hline No & 717 & 94,47 & $650(90,66)$ & $67(9,34)$ & \\
\hline
\end{tabular}


Fernando M. Runzer-Colmenares, Adriana Viñas-Mendieta, Ruth Vela-Sosa, Alessandra Vasquez, Angie Cermeño-Maraví, Enrique Moncada-Mapelli, Ian Falvy-Bockos, Miguel Cadena-Sanabria, José F. Parodi

$\begin{array}{llllll}\text { Trastorno neurocognitivo } & & & & <0,0001 \\ \text { Sí } & 240 & 31,62 & 162(67,50) & 78(32,50) & \\ \text { No } & 519 & 68,38 & 509(98,07) & 10(1,93)\end{array}$

Análisis bivariado según diagnóstico de trastorno
neurocognitvo

Mediante el análisis bivariado para el diagnóstico de TNC se hallaron diferencias estadísticamente significativas entre los niveles de educación, para las categorías un nivel de colegio completo/incompleto $(26,58 \%)$ y técnico/superior $(48,84 \%)$; del mismo modo, en el grado de instrucción militar se encontraron diferencias en aquellos que tenían el grado subalterno
$(33,4 \%)$ en comparación al grado superior $(23,97 \%)$; se encontró una asociación menor para patologías como sobrepeso/obesidad $(39,77 \%)$, enfermedad renal crónica $(48,47 \%)$ y enfermedad cerebrovascular $(88,10)$; del mismo modo no se presentó una asociación significativa para patologías como hipertensión arterial $(p=0,6)$ y depresión $(p=0,1)$. En los antecedentes como hábito tabáquico $(\mathrm{p}=<0,0001)$, sedentarismo $(p=<0,0001)$ y submarinismo $(p=<0,0001)$ se encontró una asociación significativa para TNC (Tabla 2).

Tabla 2. Análisis bivariado según diagnóstico de trastorno neurocognitivo $(n=1020)$

\begin{tabular}{|c|c|c|c|}
\hline Variables & $\begin{array}{l}\text { Cognitivamente sanos } \\
\mathrm{n}=519(68,38 \%)\end{array}$ & $\begin{array}{l}\text { Trastorno Neurocognitivo } \\
n=240(31,62 \%)\end{array}$ & Valor de $\mathrm{p}$ \\
\hline \multicolumn{3}{|l|}{ Edad } & 0,004 \\
\hline 60 - 70 años & $118(79,19)$ & $31(20,81)$ & \\
\hline $71-80$ años & $206(63,98)$ & $116(36,02)$ & \\
\hline$>80$ años & $195(67,71)$ & $93(32,29)$ & \\
\hline \multicolumn{3}{|l|}{ Educación } & $<0,0001$ \\
\hline $\begin{array}{l}\text { Colegio completo/ } \\
\text { incompleto }\end{array}$ & $431(73,42)$ & $156(26,58)$ & \\
\hline Técnica/superior & $88(51,16)$ & $84(48,84)$ & \\
\hline \multicolumn{3}{|l|}{ Grado militar } & 0,027 \\
\hline Subalterno & $408(66,56)$ & $205(33,44)$ & \\
\hline Superior & $111(76,03)$ & $35(23,97)$ & \\
\hline \multicolumn{3}{|c|}{ Diabetes mellitus tipo 2} & 0,5 \\
\hline Sí & $84(65,63)$ & $44(34,38)$ & \\
\hline No & $435(68,94)$ & $196(31,06)$ & \\
\hline \multicolumn{3}{|c|}{ Enfermedad renal crónica } & $<0,0001$ \\
\hline Sí & $84(51,53)$ & $79(48,47)$ & \\
\hline No & $435(72,99)$ & $161(27,01)$ & \\
\hline \multicolumn{3}{|l|}{ Hipertensión arterial } & 0,6 \\
\hline Sí & $308(69,06)$ & $138(30,94)$ & \\
\hline No & $211(67,41)$ & $102(32,59)$ & \\
\hline \multicolumn{3}{|l|}{ Depresión } & 0,1 \\
\hline Sí & $111(73,51)$ & $40(26,49)$ & \\
\hline No & $408(67,11)$ & $200(32,89)$ & \\
\hline \multicolumn{3}{|l|}{ Sobrepeso/Obesidad } & 0,009 \\
\hline Sí & $103(60,23)$ & $68(39,77)$ & \\
\hline No & $416(70,75)$ & $172(29,25)$ & \\
\hline \multicolumn{3}{|c|}{ Antecedente de hábito tabáquico } & $<0,0001$ \\
\hline Sí & $380(77,87)$ & $108(22,13)$ & \\
\hline No & $139(51,29)$ & $132(48,71)$ & \\
\hline
\end{tabular}




\begin{tabular}{|c|c|c|c|}
\hline \multicolumn{3}{|l|}{ Sedentarismo } & \multirow[t]{2}{*}{$<0,0001$} \\
\hline Sí & $78(48,15)$ & $84(51,85)$ & \\
\hline No & $441(73,87)$ & $156(26,13)$ & \\
\hline \multicolumn{3}{|c|}{ Enfermedad cerebrovascular } & $<0,0001$ \\
\hline Sí & $5(11,90)$ & $37(88,10)$ & \\
\hline No & $514(71,69)$ & $203(28,31)$ & \\
\hline \multicolumn{3}{|c|}{ Especialidad durante servicio militar } & $<0,0001$ \\
\hline Submarinismo & $10(11,36)$ & $78(88,64)$ & \\
\hline Otros & $509(75,86)$ & $162(24,14)$ & \\
\hline
\end{tabular}

Regresión de Poisson: Factores asociados a trastorno neurocognitivo

En el análisis ajustado de regresión de Poisson, los factores asociados al TNC fueron los siguientes: el rango de edad de 71 - 80 años de edad (RP=1,69; IC95\%: 1,12 - 2,54), grado militar superior (RP=0,62; IC95\%: 0,43 - 0,89), enfermedad cerebro vascular $(\mathrm{RP}=1,48$; IC95 \%: 1,01 - 2,23) y especialidad de submarinismo (RP=2,36; IC95 \%: 1,64 - 3,39) (Tabla 3).

Tabla 3. Regresión de Poisson: Factores asociados a trastorno neurocognitivo $(n=1020)$

\begin{tabular}{|c|c|c|}
\hline \multicolumn{3}{|l|}{ Edad } \\
\hline 60 - 70 años & Referencia & Referencia \\
\hline $71-80$ años & $1,73(1,17-2,57)$ & $1,69(1,12-2,54)$ \\
\hline$>80$ años & $1,55(1,03-2,33)$ & $1,37(0,90-2,08)$ \\
\hline \multicolumn{3}{|l|}{ Educación } \\
\hline Colegio completo/incompleto & Referencia & Referencia \\
\hline Técnica/superior & $0,54(0,42-0,71)$ & $1,06(0,77-2,08)$ \\
\hline \multicolumn{3}{|l|}{ Grado Militar } \\
\hline Subalterno & Referencia & Referencia \\
\hline Superior & $0,72(0,50-1,03)$ & $0,62(0,43-0,89)$ \\
\hline \multicolumn{3}{|l|}{ Enfermedad Renal Crónica } \\
\hline Sí & $1,79(1,37-2,35)$ & $1,26(0,94-1,70)$ \\
\hline No & Referencia & Referencia \\
\hline \multicolumn{3}{|l|}{ Sobrepeso/Obesidad } \\
\hline Sí & $1,36(1,03-1,80)$ & $1,30(0,97-1,74)$ \\
\hline No & Referencia & Referencia \\
\hline \multicolumn{3}{|l|}{$\begin{array}{l}\text { Antecedente de hábito } \\
\text { tabáquico }\end{array}$} \\
\hline Sí & $0,45(0,35-1,01)$ & $0,60(0,44-1,03)$ \\
\hline No & Referencia & Referencia \\
\hline \multicolumn{3}{|l|}{ Sedentarismo } \\
\hline Sí & $1,98(1,52-2,59)$ & $1,33(0,98-1,80)$ \\
\hline No & Referencia & Referencia \\
\hline \multicolumn{3}{|l|}{ Enfermedad cerebrovascular } \\
\hline Sí & $3,11(2,19-4,42)$ & $1,48(1,01-2,23)$ \\
\hline No & Referencia & Referencia \\
\hline \multicolumn{3}{|c|}{ Especialidad durante servicio militar } \\
\hline Submarinismo & $3,67(2,80-4,81)$ & $2,36(1,64-3,39)$ \\
\hline Otros & Referencia & Referencia \\
\hline
\end{tabular}




\section{DISCUSIÓN}

El objetivo de este estudio fue explorar la asociación entre el antecedente de servicio militar como submarinista y el desarrollo de TNC en adultos mayores de la Marina de Guerra del Perú. Encontramos que existe mayor probabilidad de problemas cognitivos en marinos retirados expuestos a submarinismo en comparación a otro tipo de especialidades militares; esto se debería a la exposición a condiciones hiperbáricas de forma crónica ${ }^{(20)}$. Los TNC son causa muy común de dependencia en el adulto mayor.

Se han descrito múltiples factores de riesgo; sin embargo, hay otros poco estudiados como el submarinismo, que, según nuestros hallazgos, aumenta las probabilidades de desarrollar TNC. Este hallazgo se apoya con lo reportado en distintos estudios señalando problemas neurológicos en personas expuestas a submarinismo; Macdiarmid demostró la presencia de alteraciones en la concentración y olvidos en personas buceadoras y sin antecedentes disbáricos ${ }^{(21)}$. En Ancud, Chile, se realizó un estudio en pacientes buceadores donde se logró encontrar alteraciones de memoria ${ }^{(22)}$.

En un reporte gubernamental presentado por la Marina de Guerra de los Estados Unidos, se pudo ver la relación entre el submarinismo y diversas enfermedades tales como; neumotórax, embolias y demencia; sin embargo, no hay especificaciones sobre cuantificación de riesgo ${ }^{(23)}$. En Noruega, Todnem et al realizaron un estudio de tipo transversal cuyo objetivo fue evaluar las posibles consecuencias de buceo profundo (190-500 metros bajo el mar) donde se reportó que las afecciones más comunes fueron trastornos de concentración y parestesias en extremidades, con patrones electroencefalográficos alterados ${ }^{(24)}$. Asimismo, el mismo grupo encontró ondas lentas focales en áreas temporales y potencialidades agudas en electroencefalogramas de buceadores, traduciendo déficit neuronal probablemente debido a exposiciones a isquemia en la corteza cerebral, sin profundizar en estudios de funciones cognitivas ${ }^{(25)}$. Por otro lado, Tetzlaff et al concluyen que existe riesgo de sufrir efecto a largo plazo en el sistema nervioso central como alteración en la rapidez mental o rendimiento visual incluso en ausencia de patología neurológica concomitante en sujetos expuestos a submarinismo ${ }^{(16)}$. En 2014, Hemelryck et al encontraron relación en reducción de la memoria a corto plazo en personas expuestas a submarinismo recreacional ${ }^{(13)}$. Sin embargo, otros estudios muestran ausencia de secuelas a largo plazo si el trabajo se realiza en condiciones adecuadas ${ }^{(26)}$ y también señalan que la relación entre TNC y submarinismo aún no han sido demostrada fehacientemente ${ }^{(20)}$.

En nuestro estudio, encontramos que la población expuesta tuvo 2,36 veces más probabilidades de desarrollar TNC en comparación con personal militar retirado de actividades distintas al submarinismo. No encontramos estudios que refuercen nuestros resultados. Por otro lado, encontramos una asociación significativa entre edad y TNC, lo cual coincide con la mayoría de estudios en relación a demencias ${ }^{(27)}$.

En el presente estudio no se halló asociación entre submarinismo e hipertensión arterial, enfermedad renal crónica o diabetes mellitus; sin embargo, un estudio realizado en Corea, señala que la apnea voluntaria durante el buceo, induce a cambios cardiovasculares, incluyendo bradicardia, reducción del gasto cardíaco e hipertensión arterial ${ }^{(28-33)}$, en dicho estudio también se logró demostrar que los repetitivos cuadros de apnea por buceo, potencialmente pueden causar a largo plazo un deterioro en la función renal ${ }^{(34)}$. La evaluación neuropsicológica cobra gran importancia en los pacientes expuestos a submarinismo, ya sea por buceo recreacional u ocupacional. Existen publicaciones recientes que señalan la afectación a nivel de funciones ejecutivas evaluadas por el test de Stroop (control inhibitorio) la cual se ve comprometida con inmersiones a los $20 \mathrm{~m}$ de profundidad (30). Por otra parte, Ergen M et al (2017) han demostrado peor desempeño en test neuropsicológicos sobretodo en sujetos con exposición extensa (concerniente a memoria episódica visual hemisférica derecha y habilidades visuoconstruccionales), planteando el deterioro cognitivo como una consecuencia a largo plazo del submarinismo ${ }^{(31)}$.

El presente estudio contó con limitaciones: 1) los datos utilizados se obtuvieron de una base de datos del Centro Médico Naval, por lo que no se puede determinar con exactitud cómo fueron las condiciones de trabajo de los participantes, si tuvieron un control estricto de las presiones de gases o si contaron con el equipo adecuado, lo cual puede disminuir el grado de confiabilidad de los resultados; 2) no se cuentan con datos de tiempo inmersión ni tiempo de servicio; 3 ) adicionalmente, no se cuentan con datos para hacer análisis de tiempo para evento (regresión de (ox), que hubiese sido la regresión ideal.

En conclusión, los pacientes que han practicado el submarinismo tienen un mayor riesgo de padecer un deterioro cognitivo. Nuestros datos proporcionan más pruebas de que el submarinismo y el deterioro cognitivo se encuentran relacionados.

\section{REFERENCIAS BIBLIOGRÁFICAS}

1. Bauer UE, Briss PA, Goodman RA, Bowman BA. Prevention of chronic disease in the 21st century: elimination of the leading preventable causes of premature death and disability in the USA. Lancet. 2014;384(9937):45-52.

2. Miranda JJ, Kinra S, Casas JP, Davey Smith G, Ebrahim S. Noncommunicable diseases in low- and middle-income countries: context, determinants and health policy. Trop Med Int Health. 2008;13(10):1225-34.

3. Fried LP, Ferrucci L. Etiological role of aging in chronic diseases: from epidemiological evidence to the new Geroscience. In: Sierra F, Kohanski R, editors. Advances in Geroscience. New York: Springer;2016. p.37-51.

4. Olivera J, Tournier I. Successful ageing and multi-dimensional poverty: the case of Peru. Ageing Soc; 36(8):1690-714.

5. Prince Martin, Wimo Anders, Guerchet Maëlenn, Ali GemmaClaire, Wu Yu-Tzu,Prina Matthew, etal. Informe Mundial sobre el Alzheimer 2015. El Impacto Global de la Demencia: Análisis de prevalencia, incidencia, coste y tendencias Informe Mundial sobre el Alzheimer. Londres, Reino Unido: Asociación Internacional de Alzheimer (ADI); 2015.

6. Rodriguez JJL, Ferri CP, Acosta D, Guerra M, Huang Y, Jacob $\mathrm{K}$, et al. Prevalence of dementia in Latin America, India, and 
China: a population-based cross-sectional survey. Lancet. 2008;372(9637):464-74.

7. Instituto Nacional de Salud Mental "Honorio Delgado - Hideyo Noguchi". Estudio Epidemiológico de Salud Mental en Lima Metropolitana y Callao- Replicación 2012. Informe General. Anales de Salud Mental. 2013; 29 (1): 1-392

8. Parodi JF, Nieto-Gutierrez W, Tellez WA, Ventocilla-Gonzales I, Runzer-Colmenares FM, Taype-Rondan A. Gait speed and the appearance of neurocognitive disorders in older adults: Results of a Peruvian cohort. Rev Esp Geriatr Gerontol. 2017; 53(1).

9. Wimo A, Guerchet M, Ali G-C, Wu Y-T, Prina AM, Winblad B, et al. The worldwide costs of dementia 2015 and comparisons with 2010. Alzheimer's Dement. 2017 Jan; 13(1):1-7.

10. Bos I, Vos SJ, Frölich L, Kornhuber J, Wiltfang J, Maier W, et al. The frequency and influence of dementia risk factors in prodromal Alzheimer's disease. Neurobiol Aging. 2017;56 (numero):33-40.

11. Panza F, Solfrizzi V, Barulli MR, Santamato A, Seripa D, Pilotto A, et al. Cognitive Frailty: A Systematic Review of Epidemiological and Neurobiological Evidence of an Age-Related Clinical Condition. Rejuvenation Res. 2015;18(5):389-412.

12. Petrova N, Mehrabian S, Raycheva M, Traykov L. The frequency of cognitive impairment and dementia one year after ischemic stroke in Bulgarian patients. Meditsinski Pregl / Med Rev. 2014;50(2):49-53.

13. Hemelryck W, Germonpré P, Papadopoulou V, Rozloznik $M$, Balestra C. Long term effects of recreational SCUBA diving on higher cognitive function. Scand J Med Sci Sports. 2014;24(6):928-34.

14. Navas $A H$, Salgado $M M$, Moreno ÁM. Barotraumatismo del piloto. ¿Puede ayudar la logopedia en estos casos? REDUCA (Enfermería, Fisioter y Podol).2016;8(2):1-11.

15. Kim JW, Oh J-K, Byun Y-S, Shon O-J, Park JH, Oh HK, et al. Incidence of Avascular Necrosis of the Femoral Head After Intramedullary Nailing of Femoral Shaft Fractures: AMulticenter Retrospective Analysis of 542 Cases. Medicine. 2016;95(5):1-4.

16. Tetzlaff K, Friege L, Hutzelmann A, Reuter M, Höll D, Leplow B. Magnetic resonance signal abnormalities and neuropsychological deficits in elderly compressed-air divers. Eur Neurol. 1999;42(4):194-199.

17. Runzer-Colmenares FM, Samper-Ternent $\mathrm{R}$, Al Snih S, Ottenbacher KJ, Parodi JF, Wong R. Prevalence and factors associated with frailty among Peruvian older adults. Arch Gerontol Geriatr. 2014;58(1):69-73.

18. Lobo A, Lobo CA, Saz P, Marcos G, Día JL, De La Cámara C, et al. Re-validation of the Mini-Examen Cognoscitivo (first Spanish version of the Mini-Mental Status Examination) and population-based norms in the elderly community. Med Clin. 1999; 112(20):767-774.

19. Washburn RA, Smith KW, Jette AM, Janney CA. The Physical Activity Scale for the Elderly (PASE): development and evaluation. J Clin Epidemiol. 1993;46(2):153-162.

20. Nogales-Gaete J, Archibaldo Donoso S, Renato J, Verdugo L. Tratado de neurología Clínica. . 1a ed. Chile: Ediciones Universitaria; 2005. 738 p.

21. Macdiarmid, JI; Ross, JAS; Watt, SJ; Osman, LM; Lawson, A; Godden, D; Stephenson, R. Potential long term health effects of diving at work: health related symptoms reported by professional divers. Undersea and Hyperbaric Medical Society.

22. Calderon J. Determination of neurological sequeale by disbaric accidents in a group of divers of chiloe, chile. En: Proceedings of the Fifteenth International Congress on Hyperbaric Medicine. Barcelona: CRIS-UTH;2005.198-199.

23. Hoiberg A, Blood C. Effects of Age and Exposure on the Health Status of U.S. Navy Divers. San Diego, United States: Naval health research center; 1984. Report No: NAVHLTHRSCHC-84-3.

24. Todnem K, Nyland H, Skeidsvoll H, Svihus R, Rinck P, Kambestad BK, et al. Neurological long term consequences of deep diving. $\mathrm{Br} \mathrm{J}$ Ind Med. 1991;48(4):258-66.
25. Todnem K, Skeidsvoil H, Svihus R, Rinck P, Riise T, Kambestad Bk, et al. Electroencephalography, evoked potentials and MRI brain scans in saturation divers. An epidemiological study. Electroencephalogr Clin Neurophysiol. 1991;79(4):322-9.

26. Cordes P, Keil R, Bartsch T, Tetzlaff K, Reuter M, Hutzelmann $A$, et al. Neurologic outcome of controlled compressed-air diving. Neurology. 2000;55(11):1743-5.

27. Owens S. Simple Tests of Balance, Grip Strength May Predict Late-Age Dementia Risk: Neurology Now. United States: American Brain Foundation. 2016

28. Manley L. Apnoeic heart rate responses in humans. A review. Sports Med. 1990;9(5):286-310.

29. Linér $M H$, Ferrigno $M$, Lundgren $C E$. Alveolar gas exchange during simulated breath-hold diving to $20 \mathrm{~m}$. Undersea Hyperb Med J Undersea Hyperb Med Soc Inc. 1993;20(1):27-38.

30. Ferretti G. Extreme human breath-hold diving. Eur J Appl Physiol. 2001;84(4):254-71.

31. Lindholm P, Lundgren CEG. The physiology and pathophysiology of human breath-hold diving. J Appl Physiol (1985). 2009;106(1):284-92.

32. Dujic Z, Breskovic T. Impact of breath holding on cardiovascular respiratory and cerebrovascular health. Sports Med. 2012;42(6):459-72.

33. Breskovic T, Uglesic L, Zubin P, Kuch B, Kraljevic J, Zanchi J, et al. Cardiovascular changes during underwater static and dynamic breath-hold dives in trained divers. J Appl Physiol (1985). 2011;111(3):673-8.

34. Oh YJ, Jung JY, Kim SS, Chae K-S, Rhu J, Lee C. The association of kidney function with repetitive breathhold diving activities of female divers from Korea, Haenyeo. BMC Nephrol. 2017;18(1):75.

\section{Fuentes de financiamiento:}

Este artículo ha sido financiado por los autores.

\section{Conflictos de interés:}

Los autores declaran no tener ningún conflicto de interés.

\section{Correspondencia:}

Fernando M. Runzer-Colmenares

Dirección: Caminos del Inca Ave. 556, Surco 33, Lima, Perú.

Teléfono: +1 5112722500

Correo electrónico: frunzerc@usmp.pe

Recibido: 11 de setiembre de 2017 Evaluado: 13 de setiembre de 2017 Aprobado: 29 de setiembre de 2017

(c) La revista. Publicado por Universidad de San Martín de Porres, Perú. (сc) ву Licencia de Creative Commons Artículo en acceso abierto bajo términos de Licencia Creative Commons Atribución 4.0 Internacional. (http://creativecommons.org/licenses/by/4.0/)

\section{ORCID iDs}

Fernando M. Runzer-Colmenares (iD https: / / orcid.org/0000-0002-7717-8996 Adriana Viñas-Mendieta Ruth Vela-Sosa

Alessandra Vásquez Angie Cermeño-Maravi Enrique Moncada-Mapelli lan Falvy-Bockos Miguel Cadena-Sanabria José F. Parodi
(D) https: / / orcid org/0000-0002-7198-1243 https: / / orcid.org/0000-0001-5346-806X Dhttps: / /orcid.org/0000-0002-8646-4014 https: / / orcid.org/0000-0002-2893-9816 https: / / orcid.org/0000-0002-2297-0695 D https: / / orcid.org/0000-0002-7328-5292

Dhttps: / /orcid.org/0000-0001-9807-3029 https: / / orcid.org/0000-0002-0336-0584 